\title{
Alumnos en las consultas clínicas. Normas de estilo para un mejor aprovechamiento de las rotaciones clínicas de los estudiantes de medicina
}

\author{
Francesc Borrell-Carrió, Joan de Pablo-Rabasso, Ramón Pujol-Farriols, Francesc Gudiol-Munté
}

\section{Introducción}

Los estudiantes de medicina efectúan una parte de sus rotaciones en entornos como salas de hospitalización, consultas externas y centros de salud, donde comparten las experiencias clínicas con un médico al que llamaremos 'tutor'. La reforma de los planes docentes abordados por las facultades de medicina pone el acento en este tipo de prácticas $[1,2]$. El presente trabajo se enmarca en un esfuerzo de la Facultad de Medicina de la Universitat de Barcelona para hacer presente las habilidades de comunicación en la tarea docente [3,4], e indica algunas pautas para la recepción y acomodación de estos estudiantes, así como situaciones delicadas que deben ser resueltas con asertividad por parte del tutor. Seguiremos el esquema de la tabla I, que ofrecemos a modo de resumen conceptual.

\section{Estilo de comunicación}

El estilo de comunicación entre tutor y alumno viene condicionado por la actitud del tutor, predominante en esta interacción. La observación sistemática sugiere tres estilos fundamentales que podrían describirse como sigue:

- Estilo autoritario/directivo: basado en una comunicación unidireccional con poca adaptación a las características del alumno. En ocasiones, este estilo esconde cierta inseguridad del tutor ('me mantendré autoritario, no sea que empiecen a hacer peguntas y no sepa qué decir').

- Estilo indiferente/distante: centrado en exposiciones puntuales. Resulta un estilo propio de personas que piensan que no volverán probablemente a ver a estos alumnos en el resto de su vida y que no merece la pena ninguna inversión de tipo emocional; puede favorecer la pasividad del alumno.
- Estilo implicado/participativo: planteado desde la proximidad en la interacción, suele adaptarse a las necesidades del alumno. El peligro de este estilo estriba en favorecer una proximidad excesiva, que suele conllevar una pérdida de ascendencia sobre el alumno (estilo de 'colegas').

En la práctica, el estilo de cada tutor suele ser una mezcla de los tres descritos, dependiendo también del alumno que se recibe. Resulta conveniente conseguir un estilo propio en el que, sintiéndonos cómodos, percibamos una máxima eficacia docente y proporcione a todos los alumnos una similar oportunidad para interaccionar con el docente. Las notas que siguen persiguen el objetivo de facilitar esta progresión del médico tutor y conseguir una menor variabilidad en la recepción y acomodación de los alumnos en los servicios asistenciales.

\section{Recepción del alumno}

Los estudiantes suelen asignarse a un médico tutor durante períodos de varias semanas. Es conveniente que esta asignación no se altere y que no se asigne más de un alumno por tutor. El estudiante debe acudir al servicio hospitalario, consultas externas o centro de salud con bata y fonendo, y se le debe brindar un espacio para que guarde sus objetos personales. Por lo general, el estudiante se sentará al lado del tutor.

'Bienvenida/o, mi nombre es... ¿Cómo te llamas?'. Esta breve presentación, en tono cordial, suele ser suficiente para indicar nuestro interés por el estudiante. Resulta conveniente no olvidar el nombre del estudiante y procurar mencionarlo para enfatizar este vínculo inicial. También conviene mencionar los objetivos de la rotación y verificar que coinciden con los que el estudiante conoce. Este aspecto confiere seriedad a la rotación. Mencione lo que se
Departamento de Ciencias Clínicas; Campus Bellvitge (F. Borrell-Carrió, R. Pujol-Farriols, F. Gudiol-Munté) Departamento de Psiquiatría; Campus Casanovas (J. de PabloRabasso); Facultad de Medicina; Universitat de Barcelona. Barcelona, España.

F.B.C. y J.P.R. son coordinadores de la competencia transversal de comunicación de la Facultad de Medicina de la UB.

Correspondencia:

Dr. Francesc Borrell Carrió. CAP Cornellà. Bellaterra, 41. E-08940 Cornellà de Llobregat (Barcelona).

E-mail:

12902fbc@comb.cat

(c) 2011 Educación Médica 
Tabla I. Aspectos fundamentales en el acomodo del estudiante a la consulta clínica

Recepción del alumno

'Bienvenida/o, mi nombre es... ¿Cómo te llamas?'

Mencione los objetivos de la rotación

'Procura no interrumpir al paciente cuando éste hable'

'Apunta las preguntas y luego, sin el paciente, las comentamos'

Le daremos normas para 'casos especiales'

El paciente entra en la consulta

Evitar juicios ad personam: 'este paciente es un pesado'

'Ahora entrará un paciente y deberías describirme el tipo de marcha'

'Le presento a Carlos, que es estudiante de quinto de medicina y realiza sus prácticas con nosotros'

Crear oportunidades docentes: '¿le importa si el estudiantee le hace unas preguntas...?'

Tutorización directa: 'veamos cómo realizas el Apley'

El paciente abandona la consulta

Evitaremos comentarios humorísticos o de franco sarcasmo

Nos esforzaremos por acercar el mundo del paciente al estudiante

Pondremos retos: 'para mañana podrías repasar esta enfermedad'

Dar feedback al estudiante

Comience por lo positivo: lo que sí le ha gustado

Primera persona del plural de tipo inclusivo

Sea específico, no generalice

Utilice un lenguaje descriptivo, no valorativo ni culpabilizador

Manifieste que sus opiniones son subjetivas

Concentre su atención en conductas que puedan mejorarse

No haga más de tres sugerencias

Trate de reconocer sus propias emociones: ¿son apropiadas?

El estudiante acaba su rotación

'Me ha gustado mucho conocerte, si mantienes este nivel de exigencia personal progresarás en tu carrera'

'No dudes en contactar conmigo si te puedo ser de utilidad'

'Te deseo mucho éxito en la siguiente rotación'

Valorar al estudiante (Tablas II y III)

Algunos problemas más concretos

El estudiante muestra una conducta poco apropiada

El estudiante viste de manera inapropiada

El estudiante presencia una discusión con el paciente

El estudiante está disconforme con la nota de prácticas y trata de que la rectifiquemos espera del alumno: por ejemplo, realizar un trabajo a partir de un caso clínico que él o ella escoja. Conviene dar ciertas instrucciones:

- 'Procura no interrumpir al paciente cuando éste hable; si tienes preguntas sobre la clínica del paciente, tienes que ser discreto, podemos asustarlo con nuestros comentarios'.

- 'Resultaría muy adecuado que apuntaras las preguntas que se te vayan pasando por la cabeza y si te parece, cuando el paciente abandone la consulta, es el momento para que me las hagas, $i d e$ acuerdo?.

- 'Si crees que debes comunicarme algún dato con urgencia o hacerme algún comentario, podemos trasladarnos a la consulta de aquí al lado, que está vacía.

- 'En algún caso especial quizá te diga: por favor, ¿puedes ayudar a la enfermera? Querrá decir en tal caso que es mejor que me dejes solo/a con el paciente porque está emergiendo un tema delicado o confidencial'.

- En algunos casos puede ocurrir que detectemos incumplimientos horarios de los estudiantes. Puede ser conveniente añadir: 'desde mi punto de vista, la puntualidad y el cumplimiento horario es un signo de responsabilidad de la mayor importancia. Tengo entendido que os incorporáis a la práctica a las $\mathrm{X}$ horas, ¿es así? Si algún día prevés que te atrasarás, es mejor que me lo adviertas; también si algún día no puedes venir, por motivos justificados. No dejes de advertírmelo'

Esta observación puede parecer antipática, pero no debemos olvidar la máxima que 'un estudiante exigido es un estudiante valorado.' Con una frase de este tipo le estamos indicando no sólo que debe ser puntual y responsable, sino que nosotros nos tomamos en serio el rol de tutor/a. En la misma línea de reciprocidad consultaremos nuestra agenda y le advertiremos de aquellos días en los que nos ausentaremos, y qué debe hacer o a quién debe dirigirse.

\section{El paciente entra en la consulta o entramos en su habitación}

Antes de entrar en la habitación del paciente, o que un paciente entre en la consulta, puede ser conveniente compartir con el alumno algunos datos clínicos, pero mucho cuidado con realizar juicios de valor del tipo: 'es un paciente muy pesado' o 'iverás que exagerada que es!, seguro que cuando nos vea dirá tal cosa'. El alumno en general tiene una visión menos crítica de los pacientes y este tipo de comen- 
tarios pueden parecerle poco compasivos. Además, bien pudiera ocurrir que nos equivoquemos de paciente, o que éste muestre una conducta en todo opuesta a la esperada.

Resulta muy adecuado preparar una hoja de trabajo que entregaremos al alumno y en la que le propondremos aspectos de observación o de elaboración de datos semiológicos (dependiendo de la práctica clínica del tutor y de los objetivos de la rotación). Este tipo de ejercicios puede tener diversos grados de formalización, desde una simple hoja para orientar una posterior discusión hasta un ejercicio que trabajará el alumno en casa con bibliografía e incorporará a su portafolio.

Puede ser oportuno disponer de una hoja de observación como la mencionada para improvisar tareas. A modo de ejemplo: 'ahora entrará en la consulta la Sra. X. Me gustaría que rellenaras en esta hoja todos los síntomas que exprese, y si surge algún otro a lo largo de la entrevista, también lo apuntas'. O en otro ejemplo: 'ahora observarás a un paciente que tiene una enfermedad de la médula. Quisiera que apuntaras todos los detalles que observes en relación a cómo se mueve, los síntomas que refiere y la exploración neurológica que le haremos.'

Antes de que el paciente entre, explicaremos al alumno la tarea que esperamos que realice. Para ello podemos basarnos en la hoja de trabajo que mencionábamos, pero otras veces actuaremos improvisando. Una vez el paciente entra en la consulta, lo saludaremos de manera personalizada (muy recomendable darle la mano o al menos mencionarlo por el nombre) y le presentaremos al estudiante: 'le presento a Carlos, que es estudiante de quinto de medicina y realiza sus prácticas con nosotros'

Debemos crear oportunidades docentes para el estudiante, pero acordes con el grado de integración que éste tenga en el servicio. Lo ideal es tener una consulta libre en la que el estudiante pueda realizar una historia clínica y avanzar una exploración física u otras maniobras que luego supervisaremos adecuadamente. Resulta conveniente no dejarlo solo a menos que se organice una actividad docente específica, lo que debería incluir:

- Presentar al alumno al paciente como estudiante avanzado: 'le presento a N., que es un estudiante de último año de medicina que está completando su formación con nosotros.

- Exponer el objetivo de la interacción y pedir permiso al paciente: 'si le parece bien, me gustaría dejar a N. con usted unos minutos para que le haga una serie de preguntas sobre su salud'.

- El tutor debe retornar al paciente algún tipo de respuesta a la interacción con el estudiante: 'la información recogida por N. nos ha resultado muy interesante para comprender -o completarsu caso'.

Por supuesto, el estudiante no debe asumir ningún tipo de responsabilidad asistencial y debe comentar cualquier hallazgo o incidencia que se produzca en su interacción con el paciente.

He aquí varias situaciones prototípicas donde crear 'oportunidades docentes' para el estudiante:

- En consultas externas y centros de salud, a veces el paciente responde a nuestras preguntas mirando al estudiante. Esta conducta suele señalar que el paciente valora la presencia del estudiante $\mathrm{y}$, por consiguiente, no mostrará resistencias a que dicho estudiante finalice la anamnesis y exploración física (siempre con una adecuada supervisión posterior del médico responsable). Desde luego, evitaremos que parezca un castigo para el paciente: 'ya que le mira tanto, ahora se lo explica todo a él/ella'. Por el contrario: ‘ile importaría si (el nombre del estudiante) le acaba de tomar estos datos y luego nos volvemos a ver?.

- En la exploración física y determinadas exploraciones complementarias daremos instrucciones al estudiante para que nos imite y esté atento a las oportunidades: 'si ves que me pongo el guante para hacer un tacto rectal, tú detrás con el guante preparado aunque no te lo diga'. En esta eventualidad le diremos al paciente: '¿le importaría que el estudiante también le hiciera la prueba?, nos sería de mucho valor para su formación' Si vemos oposición verbal o no verbal seremos muy cuidadosos: 'no se preocupe, de todas formas le agradecemos mucho su colaboración'.

\section{El paciente abandona la consulta o nos retiramos de la habitación}

Cuando abandonamos la habitación o el paciente sale de la consulta evitaremos comentarios humorísticos o de franco sarcasmo [5]. Por ejemplo: ‘ ¡vaya pesado!', o bien, '¿te has dado cuenta del tatuaje que llevaba?'. Por el contrario, nos esforzaremos en acercar el mundo del paciente al estudiante [6,7]; por ejemplo:

- 'Tal vez te ha sorprendido que el paciente se emocionara cuando hablábamos de su hija, pero resulta que ésta padece síndrome de Down'.

- 'Te puede parecer que este paciente tiene un nivel de queja exagerado; sin embargo, debes tener en cuenta que este hombre ha trabajado desde los 14 años en ocupaciones muy duras y posteriormente en minas de carbón. 
Tabla II. Valoración de una entrevista por observación directa.

¿Cómo ha saludado el estudiante al paciente? ¿Le ha sonreído, ha mencionado su nombre, le ha dado la mano?

¿Ha averiguado los datos de salud requeridos?

¿Ha preguntado sobre el cómo, cuándo y dónde de los síntomas principales?

¿Ha sido suficientemente minucioso en la recogida de datos?

En caso de realizar la exploración física, ¿ha sido cuidadoso?

¿Se ha despedido convenientemente?

Los comentarios humorísticos, frecuentes en la práctica médica, pueden ser pertinentes en algunos casos. Cuando son compartidos con el paciente o son descriptivos de una situación ayudan a relajar la tensión y facilitan la interacción con el alumno, pero siempre deben estar presididos por el respeto hacia el paciente como un valor fundamental en el ejercicio de la medicina. En consecuencia, se debe evitar cualquier expresión peyorativa, de burla o que incluya prejuicios de género, raza o cualquier otra condición. Debemos recordar que el tutor ejerce como modelo a reproducir por el alumno y no debe colaborar a perpetuar prejuicios culturales.

En ocasiones pondremos retos:

- 'Escribe todos los diagnósticos que se te ocurran'.

- ‘Qué quería decir el paciente cuando decía...?'.

E incluso tareas para posteriores días. Por ejemplo:

- 'Aprovecha para mirar el perfil típico de colestasis y lo comentamos mañana'.

- 'Te mando a tu correo electrónico este artículo que justamente da contestación a la pregunta que me haces. Si te parece, te lo lees y mañana lo comentamos. Sobre todo me interesa que mires el algoritmo de decisión, y si hubiera cambiado nuestra decisión de hacerle la prueba si en lugar de tener estos anticuerpos positivos hubieran salido normales'.

\section{Dar feedback al estudiante}

Resulta del máximo interés que el tutor supervise de manera directa las maniobras semiológicas y técnicas que realiza el estudiante. En general resulta más difícil hacerlo en la anamnesis, ya que el estudiante suele ponerse nervioso si se ve observado.
Aun así, casi siempre resulta posible recoger el tono emocional de esta anamnesis y otros detalles de interés. Existen algunos instrumentos para la valoración de la entrevista mediante observación directa (Tabla II) $[8,9]$.

Otros procedimientos, como la misma exploración física u otros procedimientos manuales, se pueden supervisar invitando al estudiante a que los repita una vez ha visto como los realizamos (con las limitaciones obvias de cada caso), y viceversa, tras realizarla el estudiante, nosotros la repetiremos y en su caso corregiremos. La tutorización directa de las habilidades clínicas tendrá efectos duraderos en los hábitos del estudiante a condición de seguir alguna sencillas recomendaciones [10] (que sin embargo no suelen realizarse [11]):

- Comience por lo positivo: lo que sí le ha gustado.

- Use una primera persona del plural de tipo inclusivo: 'nosotros lo que ahora pretendemos con esta maniobra exploratoria es...'

- Sea específico, no generalice.

- Utilice un lenguaje descriptivo, no valorativo y mucho menos culpabilizador.

- Manifieste que sus opiniones son subjetivas.

- Concentre su atención en conductas que puedan mejorarse, sea posibilista.

- No haga más de tres sugerencias.

- Trate de reconocer sus propias emociones y pregúntese si son apropiadas para el propósito que lo anima, es decir, conectar con el estudiante.

La situación más difícil suele ser cuando debemos llamar la atención del estudiante sobre una conducta a mejorar. Valgan a modo de ejemplos:

- Ejemplo 1. 'Pienso que estás aprovechando mucho esta rotación y te quería felicitar. Muestras interés en los protocolos de tratamiento y esto es positivo. Tengo una duda en relación al horario..., ¿a qué hora deberías estar aquí?. Mensaje clave: la puntualidad es un signo de responsabilidad.

- Ejemplo 2. 'Eres muy cordial con los pacientes, pero me he fijado que sueles tratar a todos los pacientes de tú. En general, a los pacientes jóvenes les gusta, pero a los de más edad puede disgustarles.' Mensaje clave: evita tutear a personas de mayor edad, aunque desde luego me puedes tutear a mi sin ningún problema, e incluso a otros colegas, sobre todo si te invitan a hacerlo.

\section{El estudiante acaba su rotación}

El final de la rotación suele ser muy variable. En ocasiones, el estudiante se despide entre visita y visita y 
ya no le veremos más. Otras veces, varios estudiantes que finalizan la rotación obsequian a los tutores con un refrigerio en señal de agradecimiento. Siempre que podamos es interesante organizar la actividad de manera que incorpore algún tipo de cierre de las prácticas, con al menos unos minutos destinados a escuchar la valoración que los estudiantes hacen de la actividad y una exposición del tutor con un resumen de sus impresiones en relación con los objetivos docentes. En todo caso procuraremos decirles:

- 'Me ha gustado mucho conocerte; si mantienes este nivel de exigencia personal progresarás en tu carrera'.

- 'No dudes en contactar conmigo si te puedo ser de utilidad'.

- 'Te deseo mucho éxito en la siguiente rotación'.

En ocasiones, el estudiante nos pregunta por la nota que le pondremos. Constituye una situación embarazosa para la cual recomendamos los siguientes mensajes:

- 'La nota no la pongo yo solo, siempre es una tarea que comento con más compañeros'.

- 'De todas formas, te puedo adelantar que será buena en la medida en que también has tomado mucho interés', o bien, 'sin embargo puedes mejorar si en la próxima rotación haces lo siguiente...

\section{La difícil tarea de valorar con justicia al estudiante}

Valoraremos al estudiante en función de rasgos objetivos que hayamos observado. Sin embargo, la relación establecida influye y sesga - para bien o para mal- los componentes mas técnicos de la evaluación, por lo que en la medida en que esta relación se valore como muy positiva, nos pondrá en alerta acerca de nuestra capacidad para ser objetivos.

La conducta que valoraremos será la específicamente profesional: trato respetuoso, cordial y empático con el paciente y el equipo, puntualidad, cumplimiento de tareas, y franqueza y sencillez en el trato.

La actitud a valorar será la predisposición a ayudar al tutor, a los otros compañeros de rotación, el interés mostrado por el paciente y por su sufrimiento (no solo su interés por las características de la enfermedad), y las ganas de aprender cosas nuevas.

Las habilidades a valorar dependerán de cada rotación, pero de manera general consideraremos: habilidades clínica básicas (anamnesis, exploración física, razonamiento) y técnicas concretas. Por supuesto, no valoraremos que inicien su rotación sabiendo estas habilidades, sino el progreso que se
Tabla III. Valoración del estudiante al final de la rotación.

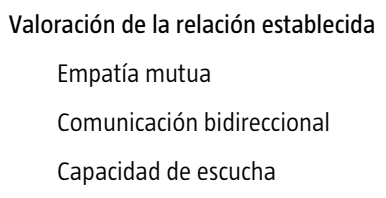

Valoración de las habilidades

Habilidades clínica básicas (anamnesis, exploración física, razonamiento) Técnicas concretas

Valoración de los conocimientos

Específicos de cada rotación

produce durante la rotación. Parecido comentario aplicaremos a la valoración de conocimientos. Los alumnos más habladores pueden hacer ostentación continua de sus saberes y los más reservados pueden parecer ignorantes.

Un ejemplo de criterios de valoración puede consultarse en la tabla III.

\section{Algunos problemas más concretos}

\section{El estudiante muestra una conducta poco apropiada}

\section{El estudiante llega sistemáticamente tarde}

Dosificaremos la gravedad del mensaje empezando por una charla en la línea del ejemplo 1 (véase más arriba). Si a pesar de esta advertencia prosigue con un reiterado incumplimiento horario, daremos noticia de ello al responsable de las prácticas para que repercuta en su nota.

\section{El estudiante está poco atento}

Posiblemente el problema sea más nuestro que del 
Tabla IV. Situaciones en las que de manera típica se producen discusiones entre paciente y medico tutor.

Por cuestiones organizativas

Retraso en la hora de visita

Retrasos en la lista de espera

Problemas acaecidos en otros servicios o con otros médicos

Por malos resultados asistenciales

Retraso diagnóstico

Error en la interpretación de un síntoma,

signo o prueba (a criterio del paciente)

Fracaso terapéutico

Por desacuerdos con una decisión institucional, de equipo o del tutor

Denegación de una prueba o de un tratamiento

Alta precipitada con posteriores secuelas

Informe clínico insatisfactorio para el paciente

estudiante, aunque también podría ocurrir que el estudiante no desee proyectar su futuro profesional en el campo de la clínica. Una charla con el estudiante en la que nos informe de cómo vive estas prácticas nos será de utilidad.

\section{El estudiante interrumpe al tutor cuando éste habla con el paciente}

En el momento en que eso ocurre no diremos nada al estudiante, pero una vez el paciente se ha retirado podemos decirle algo similar a lo siguiente: 'tus comentarios estaban cargados de buenas intenciones, pero suele suceder que en estas entrevistas el paciente está más concentrado y asimila mejor la información si solo le habla una persona; de todas maneras, si crees que me olvido de decirle algo importante, me lo puedes escribir en un papel y me será de mucha utilidad'.

\section{El estudiante realiza comentarios poco pertinentes al paciente}

La intervención anterior puede ser apropiada. Si el consejo es claramente perjudicial trataremos de reconducirlo con el paciente sin darle mayor importancia. Por ejemplo:

- Estudiante: 'Para esta lesión de la piel conviene tomar el sol'.

- Tutor: 'Probablemente estás pensando en otro tipo de lesión. (Dirigiéndose al paciente) Para esta lesión lo mejor es que no le toque el sol.'
Y si el estudiante insiste (poco probable): 'bien, (dirigiéndonos al estudiante) luego lo comentamos con más tiempo, pero usted (dirigiéndonos al paciente) tenga en cuenta que no debe tomar el sol'.

Una vez se ha retirado el paciente revisaremos la pertinencia del consejo sin dar mayor importancia al incidente, pero reiteraremos la norma de que no dé consejos al paciente.

\section{El estudiante viste de manera inapropiada o muestra signos de escasa higiene}

Una intervención podría ser: 'respeto tu manera de vestir, pero estos brazaletes deberías guardarlos en la taquilla, pueden contaminarse fácilmente e ir pasando los gérmenes de habitación en habitación. $\mathrm{O}$ también: 'es aconsejable que cada vez que me veas lavar las manos, me imites'

\section{El estudiante presencia una discusión entre el tutor y un paciente}

En general, la conflictividad viene mediada por alguna de las situaciones resumidas en la tabla IV. Si el tutor es ducho en habilidades de comunicación y -de manera mas concreta- en resolución de conflictos y negociación, será también una oportunidad para el aprendizaje [12,13]. En caso contrario es mejor que ante esta situación conflictiva el tutor sugiera al estudiante que se retire a otra consulta. Esta recomendación también será muy oportuna ante pacientes que de manera sistemática muestran conductas agresivas o sádicas, y que aprovechan la presencia de un estudiante para avergonzar al médico tutor o cuanto menos afear su conducta previa (por idónea que ésta haya sido, no olvidemos que hay algunos pacientes con graves trastornos de la personalidad) $[14,15]$.

\section{El estudiante está disconforme con la nota de prácticas y trata de que el tutor la rectifique}

Nunca cederemos a una petición de este tipo, a menos que se haya producido una confusión y hayamos valorado de manera equivocada al estudiante. La respuesta general a una petición de este tipo será: 'es encomiable tu interés por la asignatura. Lo que haré será lo siguiente: repasaré las notas que tomé de tu rotación y si fuera necesario lo comentaré con otros compañeros. Mi decisión final se la comunicaré al responsable de las prácticas. De todas maneras, ten en cuenta que es inhabitual que cambiemos el sentido de la nota porque se trata de una valoración que meditamos mucho. Espero que 
la rotación te haya servido para mejorar tus conocimientos, y eso es de verdad lo mas importante.'

\section{Conclusión}

Las prácticas en los diversos dispositivos asistenciales de hospitalización, consultas externas y centros de salud ocupan una parte muy relevante de las rotaciones de los estudiantes y de hecho constituyen la esencia de su formación en el oficio de médico. Son muchas las oportunidades de aprendizaje que se les brinda, pero también son notables los peligros. Hundert et al alertaban de los valores ocultos que transmitimos en comentarios informales, lo que él llamaba currículo 'inaparente' u 'oculto' [16]. Por dicha razón, los tutores de prácticas deben unificar algunas conductas y respuestas a las situaciones y retos más usuales, en lo que constituyen unas normas de estilo, como las apuntadas en este artículo.

\section{Bibliografía}

1. Civeira MP. El tratado de Bolonia: implicaciones en la enseñanza de medicina. Educ Med 2009; 12 (Supl 3): S1-3.

2. Steinert Y, Cruess S, Cruess R, Snell L Faculty development for teaching and evaluating professionalism: from programme design to curriculum change. Med Educ 2005; 39: 127-36.
3. Memoria para la verificación del título de grado, graduado o graduada en Medicina por la Universidad de Barcelona. Facultad de Medicina. Barcelona: UB; 2010. URL: http:// www.ub.edu.

4. De Pablo J, Borrell F. Proposta de formació en comunicació assistencial a la Facultat de Medicina de la Universitat de Barcelona. Ponència. XII Jornada de Docència dels Ensenyaments de Medicina, 27 de maig de 2010.

5. Borrell F. Compromiso con el sufrimiento, empatía, dispatía. Med Clin (Barc) 2003; 121: 785-6.

6. Stewart M. Toward a global definition of patient centred are: the patient should be the judge of patient centred care. BMJ 2001; 322: 444-5.

7. Epstein RM, Alper BS, Quill TE Communicating evidence for participatory decision making. JAMA 2004; 291: 2359-66.

8. Norcini J, Blank LL, Duffy FD, Fortna GS The Mini-CEX: a method for assessing clinical skills. Ann Intern Med 2003; 138: 476-81.

9. Borrell F. Entrevista clínica. Manual de estrategias prácticas. Barcelona: SEMFYC; 2004.

10. Van de Ridder JM, Stokking KM, McGaghie WC, Ten Cate OT. What is feedback in clinical education? Med Educ 2008; 42: 189-97.

11. Perera J, Lee N, Win K, Perera J, Wijesuriya L. Formative feedback to students: the mismatch between faculty perceptions and student expectations. Med Teach 2008; 30: 395-9.

12. Resolución de conflictos. URL: http://www.xtec.cat/ cciscart/ annexos/resoluciocooperativadeconflictes $2 . \mathrm{htm}$.

13. Sastre G, Moreno M. Resolución de conflictos y aprendizaje emocional. Barcelona: Gedisa; 2002.

14. Borrell F. Estudio del paciente conflictivo: guía para profesionales atareados. JANO 2008; 1698: 41-6.

15. Millon T. Trastornos de la personalidad. Más allá del DSM-IV. Barcelona: Masson; 1998.

16. Hundert EM, Douglas-Steele D, Bickel J. Context in medical education: the informal ethics curriculum. Med Educ 1996; 30: 353-64. 